KYUNGPOOK Math. J. 53(2013), 87-98

http://dx.doi.org/10.5666/KMJ.2013.53.1.87

\title{
Existence Results for the Nonlinear First Order Fuzzy Neu- tral Integrodifferential Equations
}

\author{
BHEEMAN RADHAKRISHNAN* \\ Department of Applied Mathematics and Computational Sciences, PSG College of \\ Technology, Coimbatore- 641 004, Tamil Nadu, India \\ e-mail : radhakrishnanb1985@gmail.com \\ Murugesan Nagarajan and Samayan Narayanamoorthy \\ Department of Applied Mathematics, Bharathiar University, Coimbatore -641 046, \\ Tamil Nadu, India \\ e-mail : mnagarajanphd@gmail.com and snmphd@yahoo.co.in
}

Abstract. In this paper, we devoted to study the existence and uniqueness of nonlinear fuzzy neutral integrodifferential equations. Moreover we study the fuzzy solution for the normal, convex, upper semicontinuous, and compactly supported interval fuzzy number. The results are obtained by using the Banach fixed-point theorem. An example is provided to illustrate the theory.

\section{Introduction}

In various fields of engineering and physics, many problems that are related to linear viscoelasticity, nonlinear elasticity have mathematical models and are described by the problems of differential or integral equations or integrodifferential equations. Integrodifferential equations are encountered in many areas of science and technology. It is well-known that the notion of aftereffect introduced in physics is very important. To model processes with aftereffect or delay it is not sufficient to employ ordinary or partial differential equations. An approach to resolve this problem is to use integrodifferential equations. Especially, one always describes a model which possesses hereditary properties by integrodifferential equations in practice. The word "fuzzy" means "vagueness". Fuzziness occurs when the boundary of a piece of information is not clear-cut. Fuzzy set have been introduced by Zadeh [21] as an extension of the classical notion of set. Classical set theory allows the membership of the elements in the set in binary terms, a bivalent condition-

* Corresponding Author.

Received September 22, 2011; revised July 26, 2012; accepted August 22, 2012.

2010 Mathematics Subject Classification: 03E72, 47H10, 34K40.

Key words and phrases: Fuzzy set, fuzzy number, neutral integrodifferential system, fuzzy solution, fixed point theorem. 
an element either belongs or does not belong to the set. Fuzzy set theory permits the gradual assessment of the membership of elements in a set, described with the aid of a membership function valued in the real unit interval $[0,1]$. Fuzzy sets have been able provide solutions to many real world problems. Fuzzy set theory is an extension of classical set theory where elements have degrees of membership.

Generally, several systems are mostly related to uncertainty and inaccuracy. The problem of inaccuracy is considered in general an exact science and that of uncertainty is considered as vague or fuzzy and accidental. The definition given here generalizes that of Aumann [2] for set-valued mappings. A differential and integral calculus for fuzzy-set-valued, shortly fuzzy-valued, mappings was developed in recent papers of Dubois and Prade [8, 9, 10] and Puri and Ralescu [20]. For fuzzy concepts, recently Diamand and Kloeden [7] established the theory of metric space of fuzzy sets. In particular, Kaleva [14] researched the fuzzy differential equations, Cauchy problem for continuous fuzzy differential equations was studied by Nieto [16], and Song et al. [18] obtained the global solutions. Park and Han [19] studied the existence and uniqueness theorem for a solution of fuzzy Volterra integral equations by using the method of successive approximation. Seikkala [17] proved the existence and uniqueness of the fuzzy solution for the following systems:

$$
\begin{aligned}
u^{\prime}(t) & =f(t, u(t)), \quad t \in[0, b], \\
u(0) & =u_{0},
\end{aligned}
$$

where $f$ is a continuous mapping from $R^{+} \times R$ into $R$ and $x_{0}$ is a fuzzy number. Recently, the above concept has been extended $t_{0}$ the integrodifferential equations by Balasubramaniam and Muralisankar [6] and Alikharni, Bahrami, Jabbari [1] proved the existence of global sloutions to nonlinear fuzzy volterra integrodifferential equations. Balachandran and Duar [4], Balachandran and Prakash [5] established the existence of perturbed fuzzy integral equations and fuzzy delay differential equations with nonlocal conditions respectively. Ding and Kandel [11] analyzed a way to combine differential equations with fuzzy sets to form a fuzzy logic systems called a fuzzy dynamical system, which can be regarded to form a fuzzy neutral functional differential equations. This paper is to investigate the existence and uniqueness of the fuzzy solution for the following nonlinear fuzzy neutral integrodifferential equations

$$
\begin{aligned}
\frac{d}{d t}(u(t)-h(t, u(t))) & =a(t) u(t)+\int_{0}^{t} k(t, s, u(s)) d s+f(t, u(t)), \quad t \in J=[0, b], \\
u(0) & =u_{0},
\end{aligned}
$$

where $a: J \rightarrow E_{N}$ is a fuzzy coefficient, $E_{N}$ is the set of all upper semicontinuous convex normal fuzzy numbers with bounded $\alpha$ - level intervals, $f: J \times E_{N} \rightarrow E_{N}$, $h: J \times E_{N} \rightarrow E_{N}$ and $k: J \times J \times E_{N} \rightarrow E_{N}$ are nonlinear continuous functions. 
Neutral differential equations arise in many areas of applied mathematics and for this reason these equations have received much attention during the last few decades $[3,12]$. There are also a number of applications in which the delayed argument occurs in the derivative of the state variable as well as in the independent variable, as in the so called neutral differential difference equations. A neutral functional differential equation is one in which the derivatives of the past history or derivatives of functionals of the past history are involved as well as the present state of the system. A good guide to the literature for neutral functional differential equations is the book by Hale and Verduyn Lunel [13] and the references therein. Neutral integrodifferential equations occur in the study of population dynamics, compartmental systems, viscoelasticity and many other fields of science.

\section{Preliminaries}

A fuzzy subset of $\mathbb{R}^{n}$ is defined in terms of a membership function which assigns to each point $x \in \mathbb{R}^{n}$ a grade of membership in the fuzzy set. Such a membership function is denoted by

$$
u: \mathbb{R}^{n} \rightarrow[0,1] .
$$

Throughout this paper, we assume that $u$ maps $\mathbb{R}^{n}$ onto $[0,1],[u]^{0}$ is a bounded subset of $\mathbb{R}^{n}, u$ is upper semicontinuous, and $\mathrm{u}$ is fuzzy convex. We denote by $E^{n}$ the space of all fuzzy subsets $\mathrm{u}$ of $\mathbb{R}^{n}$ which are normal, fuzzy convex, and upper semicontinuous fuzzy sets with bounded supports. In particular, $E^{1}$ denotes the space of all fuzzy subsets $u$ of $\mathbb{R}$.

A fuzzy number $a$ in real line $\mathbb{R}$ is a fuzzy set characterized by a membership function $\chi_{a}$

$$
\chi_{a}: \mathbb{R} \rightarrow[0,1] .
$$

A fuzzy number $a$ is expressed as

$$
a=\int_{x \in \mathbb{R}} \frac{\chi_{a}}{x}
$$

with the understanding that $\chi_{a}(x) \in[0,1]$, represents the grade of membership of $x$ in $a$ and $\int$ denotes the union of $\frac{\chi_{a}}{x}$.

Definition 2.1. A fuzzy number $a \in \mathbb{R}$ is said to be convex if, for any real numbers $x, y, z$ in $\mathbb{R}$ with $x \leq y \leq z$,

$$
\chi_{a}(y) \geq \min \left\{\chi_{a}(x), \chi_{a}(z)\right\} .
$$

Definition 2.2. The height of a fuzzy set is the largest membership value attained by any point.

Definition 2.3. If the height of a fuzzy set equals one, then the fuzzy set is called normal. Thus, a fuzzy number $a \in \mathbb{R}$ is called normal, if the followings holds:

$$
\max _{x} \chi_{a}(x)=1 .
$$


Result 2.1. Let $E_{N}$ be the set of all upper semicontinuous convex normal fuzzy numbers with bounded $\alpha$ - level intervals (see [15]). This means that if $a \in E_{N}$, then $\alpha-$ level set

$$
[a]^{\alpha}=\{x \in \mathbb{R}: a(x) \geq \alpha, 0 \leq \alpha \leq 1\},
$$

is a closed bounded interval, which we denote by

$$
[a]^{\alpha}=\left[a_{q}^{\alpha}, a_{r}^{\alpha}\right]
$$

and there exists a $t_{0} \in \mathbb{R}$ such that $a\left(t_{0}\right)=1$.

Result 2.2. Two fuzzy numbers $a$ and $b$ are called equal $a=b$, if $\chi_{a}(x)=\chi_{b}(x)$, for all $x \in \mathbb{R}$. It follows that

$$
a=b \Leftrightarrow[a]^{\alpha}=[b]^{\alpha}, \text { for all } \alpha \in(0,1] .
$$

Result 2.3. A fuzzy number a may be decomposed into its level sets through the resolution identity

$$
a=\int_{0}^{1} \alpha[a]^{\alpha},
$$

where $\alpha[a]^{\alpha}$ is the product of a scalar $\alpha$ with the set $[a]^{\alpha}$ and $\int$ is the union of $[a]^{\alpha}$ with $\alpha$ ranging from 0 to 1.

Definition 2.4. The support of a fuzzy set $A$ in the universal set $U$ is a crisp set that contains all the elements of $U$ that have nonzero membership values in $A$, that is,

$$
\operatorname{supp}(A)=\left\{x \in U: \chi_{a}(x)>0\right\},
$$

where $\operatorname{supp}(A)$ denotes the support of fuzzy set $A$. Hence the support $\Gamma_{a}$ of a fuzzy number $a$ is defined, as a special case of level set, by the following:

$$
\Gamma_{a}=\left\{x: \chi_{a}(x)>0\right\} .
$$

Definition 2.5. A fuzzy number $a \in \mathbb{R}$ is said to be positive if $0<a_{1}<a_{2}$ holds for the support $\Gamma_{a}=\left[a_{1}, a_{2}\right]$ of a, that is, $\Gamma_{a}$ is in the positive real line. Similarly, $a$ is called negative if $a_{1} \leq a_{2}<0$ and zero if $a_{1} \leq 0 \leq a_{2}$.

Lemma 2.1([17]). If $a, b \in E_{N}$, then for $\alpha \in(0,1]$,

$$
\begin{gathered}
{[a+b]^{\alpha}=\left[a_{q}^{\alpha}+b_{q}^{\alpha}, a_{r}^{\alpha}+b_{r}^{\alpha}\right],} \\
{[a b]^{\alpha}=\left[\min \left\{a_{i}^{\alpha} b_{i}^{\alpha}\right\}, \max \left\{a_{i}^{\alpha} b_{i}^{\alpha}\right\}\right], \quad(i, j=q, r),} \\
{[a-b]^{\alpha}=\left[a_{q}^{\alpha}-b_{q}^{\alpha}, a_{r}^{\alpha}-b_{r}^{\alpha}\right] .}
\end{gathered}
$$


Lemma 2.2([17]). Let $\left[a_{q}^{\alpha}, a_{r}^{\alpha}\right], 0<\alpha \leq 1$, be a given family of nonempty intervals. If

$$
\begin{gathered}
{\left[a_{q}^{\beta}, a_{r}^{\beta}\right] \subset\left[a_{q}^{\alpha}, a_{r}^{\alpha}\right], \text { for } 0<\alpha \leq \beta,} \\
{\left[\lim _{k \rightarrow \infty} a_{q}^{\alpha_{k}}, \lim _{k \rightarrow \infty} a_{r}^{\alpha_{k}}\right]=\left[a_{q}^{\alpha}, a_{r}^{\alpha}\right],}
\end{gathered}
$$

whenever $\left(\alpha_{k}\right)$ is nondecreasing sequence converting to $\alpha \in(0,1]$, then the family $\left[a_{q}^{\alpha}, a_{r}^{\alpha}\right], 0<\alpha \leq 1$, are the $\alpha$-level sets of a fuzzy number $a \in E_{N}$. Let $x$ be a point in $\mathbb{R}^{n}$ and $\mathrm{A}$ be a nonempty subsets of $\mathbb{R}^{n}$. We define the Hausdroff separation of B from A by

$$
d(x, A)=\inf \{\|x-a\|: a \in A\} .
$$

Now let $A$ and $B$ be nonempty subsets of $\mathbb{R}^{n}$. We define the Hausdroff separation of $B$ from $A$ by

$$
d_{H}^{*}(B, A)=\sup \{d(b, A): b \in B\} .
$$

In general,

$$
d_{H}^{*}(A, B) \neq d_{H}^{*}(B, A) .
$$

We define the Hausdroff distance between nonempty subsets of $A$ and $B$ of $\mathbb{R}^{n}$ by

$$
d_{H}(A, B)=\max \left\{d_{H}^{*}(A, B), d_{H}^{*}(B, A)\right\} .
$$

This is now symmetric in $A$ and $B$. Consequently,

(a) $d_{H}(A, B) \geq 0$ with $d_{H}(A, B)=0$ if and only if $\bar{A}=\bar{B}$;

(b) $d_{H}(A, B)=d_{H}(B, A)$;

(c) $d_{H}(A, B) \leq d_{H}(A, C)+d_{H}(C, B)$;

for any nonempty subsets of $A, B$ and $C$ of $\mathbb{R}^{n}$. The Hausdroff distance is a metric, the Hausdroff metric.

The supremum metric $d_{\infty}$ on $E^{n}$ is defined by

$$
d_{\infty}(u, v)=\sup \left\{d_{H}\left([u]^{\alpha},[v]^{\alpha}\right): \alpha \in(0,1]\right\}, \text { for all } u, v \in E^{n},
$$

and is obviously metric on $E^{n}$.

The supremum metric $H_{1}$ on $\mathcal{C}\left(J, E^{n}\right)$ is defined by

$$
H_{1}(x, y)=\sup \left\{d_{\infty}(x(t), y(t): t \in J), \text { for all } x, y \in \mathcal{C}\left(J: E^{n}\right)\right\} .
$$

\section{Existence and uniqueness of fuzzy solution}

In this section, we consider the existence and uniqueness of the fuzzy solution for nonlinear fuzzy neutral integrodifferential equations of the form

(3.1) $\frac{d}{d t}\left(u(t)-h(t, u(t))=a(t) u(t)+\int_{0}^{t} k(t, s, u(s)) d s+f(t, u(t)), t \in[0, b]\right.$,

$$
u(0)=u_{0},
$$


where $a: J \rightarrow E_{N}$ is a fuzzy coefficient, $E_{N}$ is the set of all upper semicontinuous convex normal fuzzy numbers with bounded $\alpha$ - level intervals, inhomogeneous $f: J \times E_{N} \rightarrow E_{N}, h: J \times E_{N} \rightarrow E_{N}$ and $k: J \times J \times E_{N} \rightarrow E_{N}$ are nonlinear continuous functions and satisfy a global Lipschitz condition, that is, there exist finite constants $\delta_{1}, \delta_{2}, \delta_{3}$ such that

$$
\begin{aligned}
d_{H}\left(\left[f\left(s, u_{1}(s)\right)\right]^{\alpha},\left[f\left(s, u_{2}(s)\right)\right]^{\alpha}\right) & \left.\left.\leq \delta_{1} d_{H}\left(\left[u_{1}(s)\right)\right]^{\alpha},\left[u_{2}(s)\right)\right]^{\alpha}\right), \\
d_{H}\left(\left[k\left(t, s, u_{1}(s)\right)\right]^{\alpha},\left[k\left(t, s, u_{2}(s)\right)\right]^{\alpha}\right) & \left.\left.\leq \delta_{2} d_{H}\left(\left[u_{1}(s)\right)\right]^{\alpha},\left[u_{2}(s)\right)\right]^{\alpha}\right), \\
d_{H}\left(\left[h\left(s, u_{1}(s)\right)\right]^{\alpha},\left[h\left(s, u_{2}(s)\right)\right]^{\alpha}\right) & \left.\left.\leq \delta_{3} d_{H}\left(\left[u_{1}(s)\right)\right]^{\alpha},\left[u_{2}(s)\right)\right]^{\alpha}\right),
\end{aligned}
$$

for all $u_{1}(t), u_{2}(t) \in E_{N}$. A mapping $u: J \rightarrow E_{N}$ of a fuzzy process $u$, then

$$
\left[u^{\prime}(t)\right]^{\alpha}=\left[\left(u_{q}^{\alpha}\right)^{\prime},\left(u_{r}^{\alpha}\right)^{\prime}\right], \quad 0<\alpha \leq 1 .
$$

The fuzzy integral

$$
\int_{a}^{b} u(t) d t, \quad a, b \in J
$$

is defined by $\int_{a}^{b}[u(t)]^{\alpha}=\left[\int_{a}^{b} u_{q}^{\alpha}, \int_{a}^{b} u_{r}^{\alpha}\right]$ provided Lebesgue integrals on the right exist.

Theorem 3.1. Let $b>0, f, g$ and $k$ satisfy a global Lipschitz condition, for every $u_{0} \in E_{N}$, then the fuzzy neutral integrodifferential equation has a unique solution $u \in \mathfrak{C}\left(J, E_{N}\right)$.

Proof. For each $u(t) \in E_{N}, t \in J$.

$$
\begin{aligned}
\left(\mathcal{F}_{0} u(t)\right)= & S(t)\left[u_{0}-h\left(0, u_{0}\right)\right]+h(t, u(t))+\int_{0}^{t} S(t-s) h(s, u(s)) d s \\
& +\int_{0}^{t} S(t-s)\left(\int_{0}^{s} k(s, r, u(r)) d r\right) d s+\int_{0}^{t} S(t-s) f(s, u(s)) d s
\end{aligned}
$$

where $S(t)$ is a fuzzy number and

$$
\begin{aligned}
{[S(t)]^{\alpha} } & =\left[S_{q}^{\alpha}, S_{r}^{\alpha}\right] \\
& =\left[\exp \left\{\int_{0}^{t} a_{q}^{\alpha}(s)\right\}, \exp \left\{\int_{0}^{t} a_{r}^{\alpha}(s)\right\}\right],
\end{aligned}
$$

and $S_{i}^{\alpha}(t)(i=q, r)$ is continuous. That is, there exists a constant $\delta>0$ such that $\left|S_{i}^{\alpha}(t)\right| \leq \delta$, for all $t \in J$. Thus $\mathcal{F}_{0} u: J \rightarrow E_{N}$ is continuous, $\mathcal{F}_{0}: \mathcal{C}\left(J, E_{N}\right) \rightarrow$ 
$\mathcal{C}\left(J, E_{N}\right)$. For $u_{1}, u_{2} \in \mathcal{C}\left(J, E_{N}\right)$, we have

$$
\begin{aligned}
& \left.d_{H}\left(\left[\left(\mathcal{F}_{0}\left(u_{1}\right)(t)\right)\right]^{\alpha},\left(\mathcal{F}_{0}\left(u_{2}\right)(t)\right)\right]^{\alpha}\right) \\
& =d_{H}\left(\left[S(t)\left[u_{0}-h\left(0, u_{0}\right)\right]+h\left(t, u_{1}(t)\right)+\int_{0}^{t} S(t-s) h\left(s, u_{1}(s)\right) d s\right.\right. \\
& \left.+\int_{0}^{t} S(t-s)\left(\int_{0}^{s} k\left(s, r, u_{1}(r)\right) d r\right) d s+\int_{0}^{t} S(t-s) f\left(s, u_{1}(s)\right) d s\right]^{\alpha}, \\
& {\left[S(t)\left[u_{0}-h\left(0, u_{0}\right)\right]+h\left(t, u_{2}(t)\right)+\int_{0}^{t} S(t-s) h\left(s, u_{2}(s)\right) d s\right.} \\
& \left.\left.+\int_{0}^{t} S(t-s)\left(\int_{0}^{s} k\left(s, r, u_{2}(r)\right) d r\right) d s+\int_{0}^{t} S(t-s) f\left(s, u_{2}(s)\right) d s\right]^{\alpha}\right) \\
& =d_{H}\left(\left[S(t)\left[u_{0}-h\left(0, u_{0}\right)\right]\right]^{\alpha}+\left[h\left(t, u_{1}(t)\right)\right]^{\alpha}+\left[\int_{0}^{t} S(t-s) h\left(s, u_{1}(s)\right) d s\right]^{\alpha}\right. \\
& +\left[\int_{0}^{t} S(t-s)\left(\int_{0}^{s} k\left(s, r, u_{1}(r)\right) d r\right) d s\right]^{\alpha}+\left[\int_{0}^{t} S(t-s) f\left(s, u_{1}(s)\right) d s\right]^{\alpha}, \\
& {\left[S(t)\left[u_{0}-h\left(0, u_{0}\right)\right]\right]^{\alpha}+\left[h\left(t, u_{2}(t)\right)\right]^{\alpha}+\left[\int_{0}^{t} S(t-s) h\left(s, u_{2}(s)\right) d s\right]^{\alpha}} \\
& \left.+\left[\int_{0}^{t} S(t-s)\left(\int_{0}^{s} k\left(s, r, u_{2}(r)\right) d r\right) d s\right]^{\alpha}+\left[\int_{0}^{t} S(t-s) f\left(s, u_{2}(s)\right) d s\right]^{\alpha}\right) \\
& \leq d_{H}\left(\left[h\left(t, u_{1}(t)\right)\right]^{\alpha},\left[h\left(t, u_{2}(t)\right)\right]^{\alpha}\right) \\
& +d_{H}\left(\int_{0}^{t}\left[S(t-s) h\left(s, u_{1}(s)\right)\right]^{\alpha} d s, \int_{0}^{t}\left[S(t-s) h\left(s, u_{2}(s)\right)\right]^{\alpha} d s\right) \\
& +d_{H}\left(\int_{0}^{t}\left[S(t-s)\left(\int_{0}^{s} k\left(s, r, u_{1}(r)\right) d r\right)\right]^{\alpha} d s\right. \\
& +\int_{0}^{t}\left[S(t-s) f\left(s, u_{1}(s)\right)\right]^{\alpha} d s, \int_{0}^{t}\left[S(t-s)\left(\int_{0}^{s} k\left(s, r, u_{2}(r)\right) d r\right)\right]^{\alpha} d s \\
& \left.+\int_{0}^{t}\left[S(t-s) f\left(s, u_{2}(s)\right)\right]^{\alpha} d s\right) \\
& \leq d_{H}\left(\left[h_{q}^{\alpha}\left(t, u_{1}(t)\right), h_{r}^{\alpha}\left(t, u_{1}(t)\right)\right],\left[h_{q}^{\alpha}\left(t, u_{2}(t)\right), h_{r}^{\alpha}\left(t, u_{2}(t)\right)\right]\right) \\
& +\int_{0}^{t} d_{H}\left(\left[S_{q}^{\alpha}(t-s) h_{q}^{\alpha}\left(s, u_{1}(s)\right), S_{r}^{\alpha}(t-s) h_{r}^{\alpha}\left(s, u_{1}(s)\right)\right] d s,\right. \\
& \left.\left.\int_{0}^{t}\left[S_{q}^{\alpha}(t-s) h_{q}^{\alpha}\left(s, u_{2}(s)\right), S_{r}^{\alpha}(t-s) h_{r}^{\alpha}\left(s, u_{2}(s)\right)\right)\right] d s\right) \\
& +\int_{0}^{t} d_{H}\left(\left[S_{q}^{\alpha}(t-s)\left(\int_{0}^{s} k_{q}^{\alpha}\left(s, r, u_{1}(r)\right) d r\right), S_{r}^{\alpha}(t-s)\right.\right. \\
& \left.\left(\int_{0}^{s} k_{r}^{\alpha}\left(s, r, u_{1}(r)\right) d r\right)\right]+\left[S _ { q } ^ { \alpha } ( t - s ) \left(f_{q}^{\alpha}\left(s, r, u_{1}(r)\right), S_{r}^{\alpha}(t-s)\left(f_{r}^{\alpha}\left(s, r, u_{1}(r)\right)\right]\right.\right. \text {, } \\
& \int_{0}^{t}\left[S_{q}^{\alpha}(t-s)\left(\int_{0}^{s} k_{q}^{\alpha}\left(s, r, u_{2}(r)\right) d r\right), S_{r}^{\alpha}(t-s)\left(\int_{0}^{s} k_{r}^{\alpha}\left(s, r, u_{2}(r)\right) d r\right)\right] \\
& +\left[S_{q}^{\alpha}(t-s)\left(f_{q}^{\alpha}\left(s, r, u_{2}(r)\right), S_{r}^{\alpha}(t-s)\left(f_{r}^{\alpha}\left(s, r, u_{2}(r)\right)\right]\right) d s\right.
\end{aligned}
$$




$$
\begin{aligned}
& =\max \left(\left|h_{q}^{\alpha}\left(t, u_{1}(t)\right)-h_{q}^{\alpha}\left(t, u_{2}(t)\right)\right|,\left|h_{r}^{\alpha}\left(t, u_{1}(t)\right)-h_{r}^{\alpha}\left(t, u_{2}(t)\right)\right|\right) \\
& +\int_{0}^{t} \max \left(\mid\left[S_{q}^{\alpha}(t-s)\left(\int_{0}^{s} k_{q}^{\alpha}\left(s, r, u_{2}(r)\right) d r\right)\right.\right. \\
& \left.+S_{q}^{\alpha}(t-s)\left(f_{q}^{\alpha}\left(s, u_{2}(s)\right)+h_{q}^{\alpha}\left(t, u_{2}(t)\right)\right)\right] \\
& -\left[S_{q}^{\alpha}(t-s)\left(\int_{0}^{s} k_{q}^{\alpha}\left(s, r, u_{1}(r)\right) d r\right)+S_{q}^{\alpha}(t-s)\left(f_{q}^{\alpha}\left(s, u_{1}(s)\right)+h_{q}^{\alpha}\left(t, u_{1}(t)\right)\right)\right] \mid, \\
& \mid\left[S_{r}^{\alpha}(t-s)\left(\int_{0}^{s} k_{r}^{\alpha}\left(s, r, u_{2}(r)\right) d r\right)+S_{r}^{\alpha}(t-s)\left(f_{r}^{\alpha}\left(s, u_{2}(s)\right)+h_{r}^{\alpha}\left(t, u_{2}(t)\right)\right)\right] \\
& \left.\left.-\left[S_{r}^{\alpha}(t-s)\left(\int_{0}^{s} k_{r}^{\alpha}\left(s, r, u_{1}(r)\right) d r\right)+S_{r}^{\alpha}(t-s)\left(f_{r}^{\alpha}\left(s, u_{1}(s)\right)\right)+h_{r}^{\alpha}\left(t, u_{1}(t)\right)\right)\right] \mid\right) d s \\
& \leq \delta_{3} d_{H}\left(\left[u_{1}\right]^{\alpha},\left[u_{2}\right]^{\alpha}\right)+\delta \int_{0}^{t} \max \left(\mid\left[\left(\int_{0}^{s} k_{q}^{\alpha}\left(s, r, u_{2}(r)\right) d r\right)\right.\right. \\
& \left.+f_{q}^{\alpha}\left(s, u_{2}(s)\right)+h_{q}^{\alpha}\left(t, u_{2}(t)\right)\right]-\left[\left(\int_{0}^{s} k_{q}^{\alpha}\left(s, r, u_{1}(r)\right) d r\right)+f_{q}^{\alpha}\left(s, u_{1}(s)\right)\right. \\
& \left.+h_{q}^{\alpha}\left(t, u_{1}(t)\right)\right]|,|\left[\left(\int_{0}^{s} k_{r}^{\alpha}\left(s, r, u_{2}(r)\right) d r\right)+f_{r}^{\alpha}\left(s, u_{2}(s)\right)+h_{r}^{\alpha}\left(t, u_{2}(t)\right)\right] \\
& -\left[\left(\int_{0}^{s} k_{r}^{\alpha}\left(s, r, u_{1}(r)\right) d r\right)+\left[f_{r}^{\alpha}\left(s, u_{1}(s)\right)+h_{r}^{\alpha}\left(t, u_{1}(t)\right)\right] \mid\right) d s \\
& \leq \delta_{3} d_{H}\left(\left[u_{1}\right]^{\alpha},\left[u_{2}\right]^{\alpha}\right)+\delta \int_{0}^{t} d_{H}\left(\left[\int_{0}^{s} k\left(s, r, u_{1}(r)\right) d r\right]^{\alpha},\left[\int_{0}^{s} k\left(s, r, u_{2}(r)\right) d r\right]^{\alpha}\right) d s \\
& +\delta \int_{0}^{t} d_{H}\left(\left[f\left(s, u_{1}(s)\right)\right]^{\alpha},\left[f\left(s, u_{2}(s)\right)\right]^{\alpha}\right) d s \\
& +\delta \int_{0}^{t} d_{H}\left(\left[h\left(s, u_{1}(s)\right)\right]^{\alpha},\left[h\left(s, u_{2}(s)\right)\right]^{\alpha}\right) d s \\
& \left.\leq \delta_{3} d_{H}\left(\left[u_{1}\right]^{\alpha},\left[u_{2}\right]^{\alpha}\right)+\delta \delta_{2} b \int_{0}^{t} d_{H}\left(\left[u_{1}(s)\right)\right]^{\alpha},\left[u_{2}(r)\right]^{\alpha}\right) d s \\
& \left.\left.\left.+\delta \delta_{1} \int_{0}^{t} d_{H}\left(\left[u_{1}(s)\right)\right]^{\alpha},\left[u_{2}(s)\right)\right]^{\alpha}\right) d s+\delta \delta_{3} \int_{0}^{t} d_{H}\left(\left[u_{1}(s)\right]^{\alpha},\left[u_{2}(s)\right)\right]^{\alpha}\right) d s \\
& \leq \delta_{3} d_{H}\left(\left[u_{1}\right]^{\alpha},\left[u_{2}\right]^{\alpha}\right)+\left(\delta \delta_{2} b+\delta \delta_{1}+\delta \delta_{3}\right) \int_{0}^{t} d_{H}\left(\left[u_{1}\right]^{\alpha},\left[u_{2}\right]^{\alpha}\right) d s \\
& \leq \delta_{3} d_{H}\left(\left[u_{1}\right]^{\alpha},\left[u_{2}\right]^{\alpha}\right)+\eta \int_{0}^{t} d_{H}\left(\left[u_{1}\right]^{\alpha},\left[u_{2}\right]^{\alpha}\right) d s,
\end{aligned}
$$

where $\eta=\delta\left(\delta_{2} b+\delta_{1}+\delta_{3}\right)$. Therefore 


$$
\begin{aligned}
d_{\infty}\left(\left(\mathcal{F}_{0} u_{1}, \mathcal{F}_{0} u_{2}\right)\right. & =\sup _{t \in J} d_{H}\left(\left[\left(\mathcal{F}_{0} u_{1}\right)\right]^{\alpha},\left[\left(\mathcal{F}_{0} u_{2}\right)\right]^{\alpha}\right) \\
& \leq \delta_{3} \sup _{t \in J} d_{H}\left(\left[u_{1}\right]^{\alpha},\left[u_{2}\right]^{\alpha}\right)+\sup _{t \in J} \eta \int_{0}^{t} d_{H}\left(\left[u_{1}\right]^{\alpha},\left[u_{2}\right]^{\alpha}\right) d s \\
& \leq \delta_{3} d_{\infty}\left(\left[u_{1}\right]^{\alpha},\left[u_{2}\right]^{\alpha}\right)+\eta \int_{0}^{t} d_{\infty}\left(\left[u_{1}\right]^{\alpha},\left[u_{2}\right]^{\alpha}\right) d s .
\end{aligned}
$$

Hence

$$
\begin{aligned}
H_{1}\left(\mathcal{F}_{0} u_{1}, \mathcal{F}_{0} u_{2}\right) & =\sup _{t \in J} d_{\infty}\left(\left[u_{1}\right]^{\alpha},\left[u_{2}\right]^{\alpha}\right) \\
& \leq \delta_{3} \sup _{t \in J} d_{\infty}\left(\left[u_{1}\right]^{\alpha},\left[u_{2}\right]^{\alpha}\right)+\eta \sup _{t \in J} \int_{0}^{t} d_{\infty}\left(\left[u_{1}\right]^{\alpha},\left[u_{2}\right]^{\alpha}\right) d s \\
& \leq\left(\delta_{3}+\eta b\right) H_{1}\left(u_{1}, u_{2}\right) .
\end{aligned}
$$

We take sufficiently small $b,\left(\delta_{3}+\eta b\right)<1$. Hence, $\mathcal{F}_{0}$ is a contraction mapping. By the Banach fixed point theorem, fuzzy neutral integrodifferential equation has a unique fixed point $u \in \mathcal{C}\left(J, E_{N}\right)$.

\section{Example}

Consider the nonlinear fuzzy neutral integrodifferential equation of the form:

$$
\begin{aligned}
\left.\frac{d}{d t}\left(u(t)-\mathbf{2 t u}(t)^{2}\right)\right) & =\mathbf{2} u(t)+\mathbf{2} t u(t)^{2}+\mathbf{3} t u(t)^{2}, \quad t \in J \\
u(0) & =\mathbf{2} \in E_{N}
\end{aligned}
$$

The $\alpha$ - level set of fuzzy number $\mathbf{2}$ and $\mathbf{3}$ are

$$
\begin{gathered}
{[\mathbf{2}]^{\alpha}=[\alpha+1,3-\alpha], \text { for } \alpha \in[0,1]} \\
{[\mathbf{3}]^{\alpha}=[\alpha+2,4-\alpha], \text { for } \alpha \in[0,1] .}
\end{gathered}
$$

Let $\int_{0}^{t} k(t, s, u(s)) d s=\mathbf{2} t u(t)^{2}, f(t, u(t))=\mathbf{3} t u(t)^{2}, h(t, u(t))=\mathbf{2} t u(t)^{2}$. Then $\alpha-$ level set of $\int_{0}^{t} k(t, s, u(s)) d s=\mathbf{2} t u(t)^{2}$ is

$$
\begin{aligned}
{\left[\int_{0}^{t} k(t, s, u(s)) d s\right]^{\alpha} } & =\left[\mathbf{2} t u(t)^{2}\right]^{\alpha} \\
& =t[\mathbf{2}]^{\alpha}\left[u(t)^{2}\right]^{\alpha} \\
& =t[\alpha+1,3-\alpha]\left[\left(u_{q}^{\alpha}(t)\right)^{2},\left(u_{r}^{\alpha}(t)\right)^{2}\right] \\
& =t\left[(\alpha+1)\left(u_{q}^{\alpha}(t)\right)^{2},(3-\alpha)\left(u_{r}^{\alpha}(t)\right)^{2}\right]
\end{aligned}
$$


where $[u(t)]^{\alpha}=\left[u_{q}^{\alpha}(t), u_{r}^{\alpha}(t)\right]$ and $[2]^{\alpha}=[\alpha+1,3-\alpha]$, for $\alpha \in[0,1]$ and the $\alpha$ level set of $f(t, x(t))$ is

$$
\begin{aligned}
{[f(t, u(t))]^{\alpha} } & =\left[\mathbf{3} t u(t)^{2}\right]^{\alpha} \\
& =t[\mathbf{3}]^{\alpha}\left[u(t)^{2}\right]^{\alpha} \\
& =t\left[(\alpha+2)\left(u_{q}^{\alpha}(t)^{2}\right),(4-\alpha)\left(u_{r}^{\alpha}(t)\right)^{2}\right],
\end{aligned}
$$

where $[u(t)]^{\alpha}=\left[u_{q}^{\alpha}(t), u_{r}^{\alpha}(t)\right]$ and $[\mathbf{3}]^{\alpha}=[\alpha+2,4-\alpha]$, for $\alpha \in[0,1]$ and the $\alpha$ level set of $h(t, x(t))$ is

$$
\begin{aligned}
{[h(t, u(t))]^{\alpha} } & =\left[\mathbf{2} t u(t)^{2}\right]^{\alpha} \\
& =t[\mathbf{2}]^{\alpha}\left[u(t)^{2}\right]^{\alpha} \\
& =t[\alpha+1,3-\alpha]\left[\left(u_{q}^{\alpha}(t)\right)^{2},\left(u_{r}^{\alpha}(t)\right)^{2}\right] \\
& =t\left[(\alpha+1)\left(u_{q}^{\alpha}(t)\right)^{2},(3-\alpha)\left(u_{r}^{\alpha}(t)\right)^{2}\right] .
\end{aligned}
$$

Thus

$$
\begin{aligned}
& {\left[\int_{0}^{t} k(t, s, u(s)) d s\right]^{\alpha}+[f(t, u(t))]^{\alpha}+[h(t, u(t))]^{\alpha}} \\
& =\quad t\left[(\alpha+1)\left(u_{q}^{\alpha}(t)\right)^{2},(3-\alpha)\left(u_{r}^{\alpha}(t)\right)^{2}\right]+t\left[(\alpha+2)\left(u_{q}^{\alpha}(t)^{2}\right),(4-\alpha)\left(u_{r}^{\alpha}(t)^{2}\right)\right] \\
& \quad+t\left[(\alpha+1)\left(u_{q}^{\alpha}(t)\right)^{2},(3-\alpha)\left(u_{r}^{\alpha}(t)\right)^{2}\right] \\
& =\quad 2 t\left[(\alpha+1)\left(u_{q}^{\alpha}(t)\right)^{2},(3-\alpha)\left(u_{r}^{\alpha}(t)\right)^{2}\right]+t\left[(\alpha+2)\left(u_{q}^{\alpha}(t)^{2}\right),(4-\alpha)\left(u_{r}^{\alpha}(t)^{2}\right)\right] .
\end{aligned}
$$

Therefore

$$
\begin{aligned}
& d_{H}\left([h(t, u(t))]^{\alpha},(h(t, v(t))]^{\alpha}\right)+d_{H}\left(\left([h(t, u(t))]^{\alpha}+\left[\int_{0}^{t} k(t, s, u(s)) d s\right]^{\alpha}+[f(t, u(t))]^{\alpha}\right),\right. \\
&\left.\left([h(t, v(t))]^{\alpha}+\left[\int_{0}^{t} k(t, s, v(s)) d s\right]^{\alpha}+[f(t, v(t))]^{\alpha}\right)\right) \\
&=\quad t \max \left\{(\alpha+1)\left|u_{q}^{\alpha}(t)^{2}-v_{q}^{\alpha}(t)^{2}\right|,(3-\alpha)\left|u_{r}^{\alpha}(t)^{2}-v_{r}^{\alpha}(t)^{2}\right|\right\} \\
&+2 t \max \left\{(\alpha+1)\left|u_{q}^{\alpha}(t)^{2}-v_{q}^{\alpha}(t)^{2}\right|,(3-\alpha)\left|u_{r}^{\alpha}(t)^{2}-v_{r}^{\alpha}(t)^{2}\right|\right\} \\
&+t \max \left\{(\alpha+2)\left|u_{q}^{\alpha}(t)^{2}-v_{q}^{\alpha}(t)^{2}\right|,(4-\alpha)\left|u_{r}^{\alpha}(t)^{2}-v_{r}^{\alpha}(t)^{2}\right|\right\} \\
& \leq(3-\alpha) b\left|u_{q}^{\alpha}(t)+v_{q}^{\alpha}(t)\right| \max \left\{\left|u_{q}^{\alpha}(t)-v_{q}^{\alpha}(t)\right|,\left|u_{r}^{\alpha}(t)-v_{r}^{\alpha}(t)\right|\right\} \\
&+(4-\alpha) b\left|u_{q}^{\alpha}(t)+v_{q}^{\alpha}(t)\right| \max \left\{\left|u_{q}^{\alpha}(t)-v_{q}^{\alpha}(t)\right|,\left|u_{r}^{\alpha}(t)-v_{r}^{\alpha}(t)\right|\right\} \\
& \leq \eta d_{H}\left([u(t)]^{\alpha},[v(t)]^{\alpha}\right),
\end{aligned}
$$

where $\eta=7 b\left|u_{q}^{\alpha}(t)+v_{q}^{\alpha}(t)\right|$ and $\eta<1$. Since $f, k$ and $h$ are satisfy the global Lipschitz condition, from Theorem 3.1, the fuzzy neutral nonlinear integrodifferential equations has a unique fuzzy solution. 
Acknowledgements The authors are thankful to the anonymous referees for the improvement of this paper.

\section{References}

[1] R. Alikhani, F. Bahrami and A. Jabbari, Existence of global solutions to nonlinear Fuzzy Volterra Integrodifferential Equations, Nonlinear Anal. Theory Methods \& Appl., 75(2012), 1810-1821.

[2] R. J. Aumann, Integrals of set-valued functions, J. Math. Anal. Appl., 12(1965), 1-12.

[3] K. Balachandran and E. R. Anandhi, Neutral functional integrodifferential control systems in Banach spaces, Kybernetika, 39(2003), 359-367.

[4] K. Balachandran, J. P. Duar, Existence of solutions of perturbed fuzzy integral equations in Banach spaces, Indian J. pure Appl. Math., 21(1997), 1461-1468.

[5] K. Balachandran, P. Prakash, Existence of solutions of fuzzy delay differential equations with nonlocal conditions, J. Korean Soc. Industrial Appl. Math., 6(2002), 81-89.

[6] P. Balasubramaniam and S. Muralisankar, Existence of fuzzy solution for the Nonlinear fuzzy integrodifferential equations, Appl. Math. Letters, 14(2001), 455-462.

[7] P. Diamand and P. E. Kloeden, Metric Space of Fuzzy Sets, World Scientific, (1994).

[8] D. Dubois and H. Prade, Towards fuzzy differential calculus. Part 1: Integration of fuzzy mappings, Fuzzy Sets Sys., 8(1982), 1-17.

[9] D. Dubois and H. Prade, Towards fuzzy differential calculus. Part 2: Integration on fuzzy intervals, Fuzzy Sets Sys., 8(1982), 105-116.

[10] D. Dubois and H. Prade, Towards fuzzy differential calculus. Part 3: Differentiation, Fuzzy Sets Sys., 8(1982), 225-233.

[11] Z. Ding and A. Kandel, On the controllability of fuzzy dynamical systems (I), J. Fuzzy Math., 8(2000), 203-214.

[12] E. Hernandez and H. R. Henriquez, Impulsive partial neutral differential equations, Applied Mathematics Letters, 19(2006), 215-222.

[13] J. K. Hale and S. M. Verduyn Lunel, Introduction to Functional-Differential Equations, Springer-Verlag, New York, 1993.

[14] O. Kaleva, Fuzzy differential equations, Fuzzy Sets Sys., 24(1987), 301-317.

[15] M. Mizumpto and K. Tanaka, Some properties of Fuzzy Numbers, North Holland, (2004).

[16] J. J. Nieto, Theory of Cauchy problem for continuous fuzzy differential equations, Fuzzy Sets Sys., 102(1999), 259-262.

[17] S. Seikkala, On the fuzzy initial value problem, Fuzzy Sets Sys., 24(1987), 319-330.

[18] S. Song, L. Guo and C. Feng, Global existence solutions to fuzzy differential equations, Fuzzy Sets Sys., 115(2000), 371-376. 
[19] J. Y. Park and H. K. Han, Existence and uniqueness theorem for a solution of fuzzy Volterra integral equations, Fuzzy Sets Sys., 105(1999), 481-488.

[20] M. L. Puri and D. A. Ralescu, Differentials for fuzzy functions, J. Math. Anal. Appl., 91(1983), 552-558.

[21] L. A. Zadeh, Fuzzy Sets, Information and Control, 81(1965), 338-353. 